\title{
SYNCHROTRON INJECTORS BASED ON HIGH CHARGE STATE ION SOURCES
}

K. Prelec

February 14, 1990

ALTERNATING GRADIENT SYNCHROTRON DEPARTMIENT

BROOKHAVEN NATIONAL IABORATORY

UPTON, LONG ISLAND, NEW YORK 11973 


\section{DISCLAIMER}

This report was prepared as an account of work sponsored by an agency of the United States Government. Neither the United States Government nor any agency thereof. nor any of their employees, nor any of their contractors, subcontracion. or their empioyees, makes any warranty, express or implied. or assumes any legal liability or responsibility for the accuracy. completeness. or usefulness of any information. apparatus, product or process disclosed, or represents that its use would not infringe privately owned rights. Reference herein to any specific commercial product, process. or service by trade name, trademark, manufacturer, or otherwise, does nat necessa rily constitute or imply its endorsement, recommendation, or favoring by the United States Government or any agency, contractor of subcontractor thereof. The views and opinions of authors expressed herein do not necessarily state or reflect those of the United States Government or any agency, contractor or subcontractor thereof. 


\section{Introduction}

The performance of any injector contemplated to replace the electrostatic tandem accelerators some time in the future should evidently match or surpass the characteristics of the tandems. It is a fortunate coincidence that the performance of the BNL Tandem satisfies in most respects the requirements of the proposed collider, although originally tanciems were not built with this application in mind. Requests for heavy ion beams with parameters (ion species, intensity) suitable for injection into the rings of a heavy ion collider have appeared rather recently, at a stage when the high charge state ion sources, which in principle are capable of producing many ion species, have not yet reached such a level of performance. Therefore, consideration of such sources as part of a future injector replacing the tandem accelerators will have to rely on the extrapolation of results from existing models, developed for a different purpose. At the same time, present and future collider requirements for heavy ion beams should serve as a stimulus for the development of sources producing ions with adequate charge states and intensities. Injectors based on such sources may present a better alternative than the tandem accelerators because a higher charge-to-mass ratio of ions from the source results in a more efficient and less costly accelerator.

In this report, two candidates for a high charge state, heavy ion source will be considered: an EBIS and an ECF. Other approaches, e.g. laser ion sources, are much further away in the development of a device to be used in a synchrotron injector.

\section{Electron Beam Ion Sources (EBIS)}

In an Electron Beam Ion Source (EBIS), multiply charged ions are produced by electron impact on ions by a magnetically confined electron beam of proper energy. The ions are confined radially by the space charge of the electron beam; the axial confinement depends on the desired mode of operation, i.e. whether the source operates d.c. or in pulses. For injection into a synchrotron, only 
the pulsed mode is of interest because the source can use the interval between two extraction pulses to build up a distribution with a higher optimum charge state than in the d.c. mode. A cycle of operation in the pulsed mode consists of a short injection pulse (either atoms or singly charged ions may be injected), followed by a confinement period to build up the desired charge state distribution and ending with the expulsion of ions from the source. The shape of the axial distribution of potential varies during the cycle to assure the proper operation of the source.

As has been noted before, at present there is no system based on a high charge state heavy ion source capable of matching the expected Tandem performance as an injector for RHIC. In order to be able to scale up the yield from existing devices, it is necessary first to consider several important source parameters and their possible scaling. For an EBIS, the maximum number of positive charges $Q_{\max }$ that the trap can store equals the number of electrons in the trap $\mathcal{Q}^{-}$

$$
Q_{\max }^{+}=Q^{-}=1.05 \times 10^{13} \cdot I_{e} \cdot V_{e}^{-1 / 2} \cdot L
$$

where $I_{e}$ is the electron beam currrent in $A, V_{e}$ the electron beam voltage in $V$, and $L$ the length of the trap in meters. This relationship can also be expressed by means of perveance $P$

$$
\mathrm{Q}_{\max }^{+}=1.05 \times 10^{13} \mathrm{P} \cdot \mathrm{V}_{\mathrm{e}} \cdot \mathrm{L}
$$

In a real device, the neutralization of the electron space charge by positive ions will always be less than complete; also, the selected sharge state will only be a part of the extracted positive ion beam. Therefore, the right hand side of Eq. 1 has to be multiplied by the neutralization degree $\mathrm{K}_{1}<1\left(\mathrm{~K}_{1}=Q^{+} / \mathrm{Q}_{\max }^{+}\right)$ and by the relative charge state abundance $\mathrm{K}_{2}<1$ of the selected state $q$, to get an expression for the available number of ions $N_{q}$

$$
q \cdot N_{q}=1.05 \times 10^{13} \cdot K_{1} K_{2} I_{e} v_{e}^{-1 / 2} L
$$


There are two more parameters, electron beam current density $J$ and the interaction (or confinement) time $\tau$; their product $J \times r$ determines the evolution of the charge state distribution. Figure 1 shows the calculated values of $\mathrm{J} \times \tau$ needed to obtain $\mathrm{Ne}, \mathrm{Ar}, \mathrm{Kr}$, $X e$ and $U$ ions in charge states up to fully stripped, (1) with corresponding values of the electron beam energy.

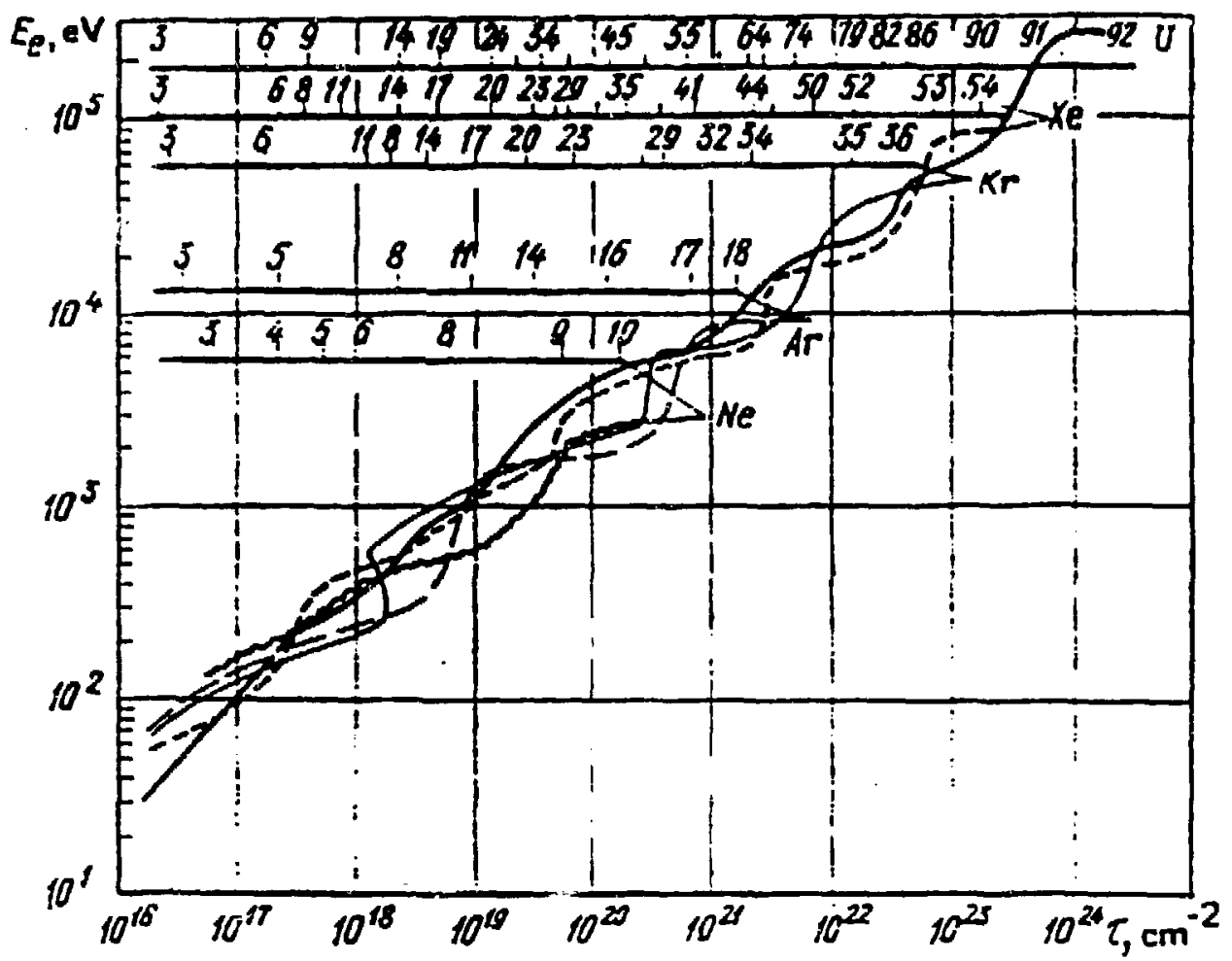

Figure 1. Calculated values of $J \tau$ needed to obtain Ne, Ar, $\mathrm{Kr}, \mathrm{Xe}$, and $\mathrm{U}$ ions of the given charge states for the corresponding energies of the bombarding electrons.

EBIS achievements, parameter by parameter, will be reviewed now before considering the requirements for a device to serve as the RHIC injector. 


\section{a. Electron Beam Current I and Current Density I}

Most EBIS sources use commercially available cathodes, that provide electron beam currents between $0.1 \mathrm{~A}$ and $0.6 \mathrm{~A}$. (2) Cathodes are usually placed outside the source magnetic field, thereby allowing a higher beam compression factor. Electron beam current densities achieved so far range from $30 \mathrm{~A} / \mathrm{cm}^{2}$ to 2000 A/ $\mathrm{cm}^{2}$. (2)

b. Electron Beam Voltage and Perveance

Electron beam energy determines the maximum possible charge state in the ion beam; depending on the application of an EBIS (e.g., ion species and charge state), the voltage values range from a few $\mathrm{kV}$ to $50 \mathrm{kV}$, the latter sufficient for a full stripping of xenon. Although the theoretical limit for the perveance of a confined flow electron beam (cathode outside the magnetic field) is $P=25 \cdot 10^{-6} \mathrm{AV}^{-3 / 2}$, practical electron guns operate with values between $0.5 \times 10^{-6}$ and $2 \times 10^{-6} \mathrm{AV}^{-3 / 2}$.

\section{c. Yield and Neutralization Degree}

The only EBIS source serving now for injection into a synchrotron is DIONE, which has been in regular operation since late 1987. From its parameters, (3) the maximum number of charges $Q_{\max }^{+}$should be $5 \times 10^{10}$; the extracted number of charges per pulse of $2 \times 10^{10}$ would correspond to a neutralization degree $k_{1}$ of 0.4 . The normal operating neutralization degree has however, been higher, up to 0.5 , at a reduced electron beam current $(0.36 \mathrm{~A}$ instead of $0.48 \mathrm{~A}$ ). It has also been observed, that the neutralization degree decreased with the confinement time, resulting in a less efficient production of highest charge states. So far, DIONE has produced and injected into an RFQ beams of ions up to krypton.

A similar EBIS is envisaged to be used as the injector into the CRYRING, a synchrotron facility in Sweden. (4), (5) Although its electron beam performance has so far been a factor of 30 lower than the design value of $\mathrm{Q}^{-}=3 \times 10^{11}$ (mostly due to technical problems), the neutralization degree was still about 0.3 , resulting in 
a value of $\mathrm{K}_{1} \mathrm{Q}^{-}$of $3 \times 10^{9}$. This source has produced fully stripped neon and argon ions and xenon ions in charge states up to 40+, with intensities up to $10^{7}$ ions per pulse. The CRYRING facility will serve in atomic, molecular, and nuclear physics. Values of the parameter $\mathrm{K}_{1} \mathrm{Q}^{-}$for other EBIS models are substantially lower, mostly because of different design requirements (e.g., a low number of very highly stripped heavy ions for atomic physics studies). (6), (7)

\section{d. Charge state Distributions, Ion Cooling}

Calculated charge state distributions in an EBIS, presented as a function of the parameter $J r$, show a continuous evolution toward the limit given by the electron beam voltage, with a gradual depletion of lower states. For a heavy ion source, a very important parameter is the relative abundance of the selected charge state. Although calculated chirge state distributions predict that one should be able to obcain at least $20 \%$ in the optimum charge state (peak of the distribution), the relative yield at the peak. is expected to improve if electrons in the outer shells are fully removed. Calculations by Becker(8) have shown that, for example, 65\% of xenon ions could be in the charge state $44+$ or $70 \%$ of lead ions in the charge state $54+$ (excluding the asymptotic value of $100 \%$ in a single charge state, predicted for very long confinement times).

The evolution of charge state distributions with confinement time has been found by several authors, mostly for lighter elements and noble gases up to xenon. (1) The general features of observed charge state spectra are the depletion of lower charge states and a shift of the optimum charge state toward higher values as the confinement time progresses. Distribution curves are rather narrow, which agrees with calculations; for example, it was possible to get $50 \%$ of gold ions in the charge state $69+,(12)$ although with rather low intensities. There is less experimental agreement concerning the location of the peak in the distribution as a function of the product $J r$. For highly stripped, lighter ions (e.g., 
argon), the peak may be one or more charge states below the value predicted by the theory; on the other hand, Donets has reported experimental results for xenon substantially better than predicted by his calculations (13) (e.g., an optimum charge state of $23+$ for a I $\tau$ of $7 \times 10^{18} \mathrm{~cm}^{-2}$; $\mathrm{cf}$. Figure 1 ), which would agree with more recent calculations.

Evaporative cooling of highly charged ions is a new development. In a conventional EBIS, as originally conceived, ions in the highest charge states may be lost due to the heating in elastic collisions with beam electrons, preventing the evolution of the charge state distribution with confinement time $\tau$ toward the theoretical limit. It has been theoretically predicted(14) and experimentally shown(12) that both the total number of high charge state ions and the confinement time (which determines their production), can be substantially increased if lighter ions in a low charge state are added into the trap. In elastic collisions with the heavier species, lighter ions are heated and continuously escape the trap, taking in this way with them the energy from the system. This process is an important improvement that may raise the practical limit of EBIS sources.

e. A state-of-the-Art EBIS for RHIC Injection

So far, the existing EBIS devices have been custom-

designed for a specific application and copying any of them would not satisfy different RHIC injection requirements. Instead, we shall consider a combination of individually achieved source parameters and project what such a hypothetical source could deliver.

If an electron gun delivering 1 A of beam current with a perveance of $2 \times 10^{-6} \mathrm{AV}^{-3 / 2}$ is used, the voltage has to be at least

$$
v=\left(2 \times 10^{-6}\right)^{-2 / 3}=5300 \mathrm{~V}
$$

Compression factors for electron beam current of 1000 have been achieved, so that with a confinement time $\tau$ of $100 \mathrm{~ms}$ 
$\mathrm{J}=1000 \mathrm{~A} / \mathrm{cm}^{2}, \mathrm{~J} \tau=100 \mathrm{As} / \mathrm{cm}^{2}$ or $\mathrm{J} \tau=6 \times 10^{20} \mathrm{~cm}^{-2}$

A practical limit on the coil length can be taken as $2 \mathrm{~m}$; the trap length could then be

$$
\mathrm{L}=1.5 \mathrm{~m}
$$

The number of electrons in the trap (or the capacity of the trap) is, therefore,

$$
Q^{-}=1.05 \times 10^{13} \mathrm{I}_{e} \mathrm{~V}^{-1 / 2} \cdot \mathrm{L}=2 \times 10^{11} \text { (negative charges) }
$$

With a neutralization degree of 50\%, such a device could store at most

$$
Q^{+}=10^{11} \text { (positive charges) }
$$

It is worthwhile noting that the values above are close to the design values of the two sources already in operation (DIONE(3), CRYEBIS(4)). An EBIS would best be followed by an RFQ and a short linac (possibly superconducting), with an equivalent voltage of about $20 \mathrm{MV}$.

The selection of the ion charge state from an EBIS depends not only on its design and operating parameters, but also on the requirements of accelerating stages up to the AGS. The first stage following the source, an RFQ, should be capable of accepting particles with a charge-to-mass ratio down to 0.2 , resulting in a relatively compact and straightforward design. The next stage, a linac, would accept a bunched ion beam from the $R F Q$, in the same charge state as selected from the source. Except for the heaviest elements $(A u, U)$, the ion charge state in the Booster should be high enough to enable an efficient full stripping before the beam is injected into the AGS. (10) The optimum charge states in the 
EBIS output ion beam have been calculated for a selected number of ion species, as a function of $J \tau$ (Figure 1 ). However, more recent calculations (8) predict lower values of $\mathrm{J} i$. The yield of ions in the optimum charge state depends primarily on the capacity of the trap, i.e., on electson beam current $I$, provided that the electron energy and the product $J \times \tau$ are high enough for the distribution to reach that optimum charge state. For the product $J \mathbf{x} \tau$, there is also a certain adjustment flexibility (e.g., increase of $\mathrm{J}$ by increasing the magnetic field or operating with longer confinement times $\tau$ ).

Several elements have been selected for consideration and comparison; they range from carbon to gold. Neon has been included as probably the heaviest element envisaged for cancer treatment, a possibility that will open with the Booster in operation. A summary of relevant parameters is shown in Table $I$. One of the advantages of an EBIS is the possibility of selecting the optimum charge state in a wide range of values by varying the operating parameters of the source; therefore, this selection does not necessarily represent the best or the final one. Several assumptions had to be made: for lighter elements, either an efficient full stripping (for carbon and nitrogen) or a stripping to the $\mathrm{K}$-shell (for neon and sulphur) can be achieved; for most other elements, a stripping to a closed-shell or subshell was selected, which may enhance the relative abundance of that state; values of the factor $k_{2}$ were taken on the basis of experimental data or calculations; for medium and heavy species, the evaporative ion cooling should be used. 


\section{TABLE I}

Charge state

Element $q / m$

0.5

0.5

0.4

0.44

0.22

0.29

0.20

0.20

0.21
$\mathrm{K}_{2}$

$\left(\begin{array}{l}\mathrm{N}_{\mathrm{q}} \\ \mathrm{10}\end{array}\right.$

0.5

$3: 3$

0.5

7.0

0.5

6.0

$14+$

0.5

3.5

$14+$

0.15

1.0

$\mathrm{Kr}$

$25+$

$26+$

$43+$

0.25

1.0

0.25

1.0

$\mathrm{Xe}$

$\mathrm{Au}$

The electron energy of $6.3 \mathrm{keV}$ is sufficient to reach any of the charge states in Table $I$, while for all ions except gold, an order of magnitude lower value of $J \times \tau$ (i.e., J $x \tau \approx 6 \times 10^{19}$ $\mathrm{cm}^{-2}$ ) would suffice.

Except for the heaviest ions (e.g., gold), the projected performance of such a state-of-the-art EBIS seems to be adequate for several modes of operation of the AGS-RHIC complex. These modes would include the fixed target Booster, AGS, and RHIC operation, the use of light and medium ions in the collider mode (especially when operating RHIC with two different mass ion beams), and tuning and studies with intensities below the full RHIC intensity. As mentioned before, such a source would be designed using existing technologies (electron guns, superconducting magnets, ultra-high vacuum) and it could serve in an operating injector and as an experimental device as well. The most important information to be gained from this device is the test of EBIS scaling laws $\left(Q^{-}=\right.$ $f\left(I_{e}\right)$ and $\left.q_{c p t}=f(J \cdot \tau)\right)$, which would serve as the basis for the possible design of the next EBIS stage. 


\section{f. Future EBIS Requirements}

Should the performance of the "state-of-the-art EBIS" be satisfactory and in accordance with scaling rules, we could consider the design of an advanced model to serve in an injector, fully replacing the Tandem. As a goal, we could define a desired injector output of $\mathrm{Au}$ ions equal to $5 \times 10^{9}$ ions per pulse and $3 \mathrm{x}$ $10^{9}$ for uranium ions; the EBIS yield of any ion lighter than gold woula be much higher and easier to achieve. Table If shows a possible selection of charge states for the two species and the required number of stored positive charges. Losses in the RFQ and the linac can be reduced to a small part of the EBIS sutput beam and the injector output should not differ much from the source output.

TABLE II

Charge State

Element

from EBIS

$q / \mathfrak{n}$

$\mathrm{K}_{2}$

$Q^{+}\left(x 10^{11}\right)$

$\mathrm{Au}$

$51+$

0.26

0.40

6.4

U

$64+$

0.27

0.40

4.8

A relatively high value of the abundance of the selected charge states (factor $K_{2}$ ) was assumed because recent calculations for lead by Becker(8) predict a high yield for ions stripped to the three inner shells. It follows that the number of negative charges in the device should be at least $Q^{-}=10^{12}$. An electron beam voltage of $20 \mathrm{keV}$ is more than sufficient to strip uranium to the three inrermost shells; from the expression for the EBIS trap capacity, assuming a maximum reasonable length of $\mathrm{L}=2 \mathrm{~m}$, it follows that

$$
I=6.7 \mathrm{~A}
$$


The perveance of such a beam is

$$
P=I V^{-3 / 2}=2.4 \times 10^{-6} \times V^{-3 / 2}
$$

By far the most difficult parameter to scale up is the electron beam current, maintaining the perveance at a high value of $2.4 x$ $10^{-6}$. The electron beam current density should be of the order of $1000 \mathrm{~A} / \mathrm{cm}^{2}$ to avoid tso long confinement times. A substantiai effort would be needed to develop such a gun and novel approaches (hollow beams; semi-immersed cathodes; multiple beams; plasma electron sources) will have to be explored. still, such a device would not oniy provide the beams up to gold, but also extend the range beyond gold to uranium.

\section{Electron Cyclotron Resonance Ion Sources (ECR-IS)}

In an electron cyclotron resonance ion source (ECR-IS), multiply charged ions are produced by impact of energetic electrons in a plasma. Electrons are selectively heated by electromagnetic waves in regions of the source where the electron cyclotron frequency equals the wave frequency. The magnetic field in modern sources of this type is of the B-minimum configuration, consisting of an axial mirrcr field with a superimposed multipoie field (usually hexapole or octopole). In such a field, there is a closed surface where the resonance condition for the waves is satisfied. ECR ion sources are in principle steady state devices, with equilibrium electron energy and ion charge state distributions, and widely used in many cyclotrons.

There are a certain number of basic relationships valid for ECR sources. In addition to the resonance condition, the wave frequency $f_{W}$ has to be higher than the plasma frequency $f_{p}$

$$
f_{w}>f_{p}=8.91 \times 10^{3} \sqrt{n_{e}}
$$


where $\mathrm{n}_{e}$ is the plasma electron density in $\mathrm{cm}^{-3}$. Any increase in $\mathrm{n}_{e}$ above the limit determined by this relationship has to be, therefore, accompanied by an increase of the wave frequency and of the magnetic field. Geller(15) has proposed several scaling laws for ECR sources, which are in a reasonable agreement with experimental data and can be used to scale up the parameters of existing devices. In distinction from EBIS sources, where a simple relationship exists between electron beam parameters and the maximum number of positive (ion) charges that can be accumulated in the device, the ECR scaling laws involve plasma parameters and are much more empirical in nature.

Two important parameters of the source output are gopt, the charge state at which the maximum ion current is extracted, and the current $I_{q, \text { opt }}$ carried by ions in this charge state. The proposed scaling rule for gopt when the $\mathrm{rf}$ is changed, is

$$
q_{o p t} \propto \log B^{3.5} \propto \log f^{3.5}
$$

If optimum charge states, $q_{1}$ and $q_{2}$, respectively, for a certain ion species are known for two rf values, $f_{1}$ and $f_{2}$, respectively, then this scaling rule would predict that the optimum charge state $q$ at some other (usually higher) frequency $f$ is

$$
q=q_{1}+\frac{q_{2}-q_{1}}{\log \left(f_{2} / f_{1}\right)} \log \left(f / f_{1}\right)
$$

The scaling is, therefore, slow--with $\log f$ only. The current in the average charge state $<q>$ scales as

$$
I_{<q>} \propto f^{2} / m_{i}
$$

In a limited ringe of frequencies, the current $I_{q \text {, opt may be taken }}$ as being prorortional to $I_{<q\rangle}$ and the same scaling rule would 
apply. The rf power will determine the electron energy; at lower levels, the electron energy is proportional to the rf power $P$, at higher levels it becomes proportional to $\mathrm{p}^{1 / 2}$ and this will be very often the case. Scaling of qopt with the rf powes (at a fixed frequency and in the non-linear regime) is

$$
\text { gopt } \propto \mathrm{p}^{1 / 6}
$$

i.e., a very slow increase with power. Scaling rules as stated above may be used to project the performance of a source if reference data are known, preferably for more than one value of the parameter in question (e.g., for $f$ or $P$ ).

Yields of ECR sources are usually quoted in units of electric currents; for the sake of comparison with both tandems and EBISbased injectors, the yields should be given in particle currents and then the number of particles available during the Booster injection interval caiculated. There are several dozens of ECR sources in operation, but only one is being used to inject heavy ions (O, S) into a syrichrotron (CERN, operation with a fixed target). Still, possible applications of ECR sources are bxoad and this fact is reflected in vigorous development programs in many laboratories around the world. Having in mind the possible application of an ECRIS in the injector for a heavy ion collider like RHIC, best results from several sources(15),(16), (17) will be compiled to represent again a state-of-the-art device. In addition to fully stripped nitrogen and helium-like neon and sulphur ions, lower charge states for some ions will also be considered. In ECR ion sources, selection of a lower charge state usually results in a much higher yield, while corresponding benefits in an

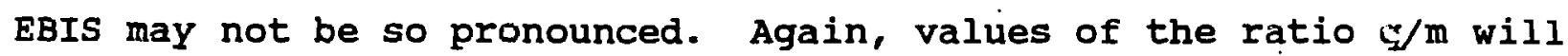
be limited to those higher than 0.2 in order to reduce the burden on next stages, the RFQ and the linac. A summary of relevant parameters is given in Table III. As it was the case before with an EBIS, there would be no stripping foil between the source and the Booster. 
TABLE III

\begin{tabular}{|c|c|c|c|c|c|}
\hline Element & $\begin{array}{l}\text { Selected } \\
\text { Charge state }\end{array}$ & $q / m$ & $\begin{array}{l}\text { Yield } \\
\text { (pps) }\end{array}$ & $\begin{array}{l}\text { Injection } \\
\text { Interval } \\
(\mu \mathrm{s})\end{array}$ & $\begin{array}{l}\text { No. of I } \\
\text { per puls } \\
\left(x 10^{9}\right)\end{array}$ \\
\hline $\mathbf{N}$ & $4+$ & 0.29 & $2 \times 10^{14}$ & 100 & 20 \\
\hline $\mathbf{N}$ & $7+$ & 0.50 & $3 \times 1.0^{12}$ & 100 & 0.3 \\
\hline $\mathrm{Ne}$ & $5+$ & 0.25 & $1.3 \times 10^{14}$ & 100 & 13 \\
\hline $\mathrm{Ne}$ & $8+$ & 0.40 & $7.8 \times 10^{13}$ & 100 & 8 \\
\hline $\mathbf{s}$ & $9+$ & 0.28 & $2.7 \times 10^{13}$ & 100 & 2.7 \\
\hline $\mathbf{S}$ & $12+$ & 0.38 & $4 \times 10^{12}$ & 100 & 0.4 \\
\hline $\mathrm{Ni}$ & $12+$ & 0.21 & $4 \times 10^{12}$ & 150 & 0.6 \\
\hline $\mathrm{Kr}$ & $17+$ & 0.2 & $10^{13}$ & 150 & 1.5 \\
\hline $\mathrm{Kr}$ & $24+$ & 0.29 & $2 \times 10^{11}$ & 150 & 0.03 \\
\hline$I$ & $26+$ & 0.20 & $4.6 \times 10^{11}$ & 200 & 0.1 \\
\hline Xe & $26+$ & 0.20 & $4 \times 1 c^{12}$ & 200 & 0.8 \\
\hline $\mathrm{Ta}$ & $37+$ & 0.20 & $10^{11}$ & 300 & 0.03 \\
\hline $\mathrm{Au}$ & $43+$ & 0.21 & $10^{11}$ & 300 & 0.03 \\
\hline$(\mathrm{U}$ & $32+$ & 0.13 & $2 \times 10^{11}$ & 400 & $0.08)$ \\
\hline
\end{tabular}

In Table III, nickel was chosen instead of copper. Comparison of Table I and Table III shows that from the point of view of a RHIC injector, the two sources are comparable for light and medium heavy ions (up to krypton), especially if a lower charge state from an ECR source is selected. However, for heavier iors, an injector based on an ECR souxce would have to operate with much lower values of the ratio $q / m$ than an EBIS source in order to achieve the required beam intensity; this will make the next stages (RFQ and linac) much larger and more expensive. 
So far, the experience with ECR sources has been limited to the operation below $18 \mathrm{GHz}$. There are several devices under development for higher frequencies reaching even into the frequency range of gyrotrons. (18) Assuming for the sake of comparison that the rf frequency of the MINIMAFIOS ECR source has bean increased to $30 \mathrm{GHz}$, scaling rule for qopt with frequency would predict higher values, as shown in Table IV.

TABIE IV

\begin{tabular}{lrccccc} 
f(GHz) & \multicolumn{2}{c}{ Ar } & \multicolumn{2}{c}{ Ta } & \multicolumn{2}{c}{$U$} \\
& qopt $q / \mathrm{m}$ & Gopt & $q / \mathrm{m}$ & qopt & $q / \mathrm{m}$ \\
10 & 8 & 0.2 & 18 & 0.1 & 18 & 0.075 \\
16.6 & 9 & 0.23 & 24 & 0.13 & 25 & 0.11 \\
30 & 10 & 0.25 & 31 & 0.17 & 33 & 0.14
\end{tabular}

Predicting the yield in such a mode of operation is equally difficult and the only way to find out is by building a device and testing it.(18) However, scaling up in frequency may pose new problems. First, the required magnetic fields scale with the frequency and above 20-30 $\mathrm{GHz}$ may require superconducting coils. While it is in principle possible to design a small source using permanent magnets, there is a minimum size limit for sextupole windings. (19) The required rf power goes up with frequency and volume of the source, so that a source operating at frequencies above $30 \mathrm{GHz}$ may require power levels of tens of $\mathrm{kW}$, which not only represents a aifficult cooling problem, but requires expensive rf power supplies. Rather slow scaling of the optimum charge state with frequency and $x f$ power seems to pose a fundamental limit for the development of an ECR source with a substantially higher optimum charge state than presently available, especially concerning the heaviest elements. 


\section{Post-Acceleration of High Charge Stcate Ion Beams}

The first stage of acceleration after the high charge state source should be a radio frequency quadrupole (RFQ), a devi.ce ideally suited for that purpose. An RFQ accepts a C.W. beam, bunches it and accelerates with an efficiency around 90\%. J. staples(22) has done a design study for a possible device accepting beams from an EBIS(20) similar to the "state-of-the-art" model described earlier. This RFQ would accept ions of any species if their $q / m$ is larger than 0.228 , having an input energy of 11 $\mathrm{keV} / \mathrm{amu}$ and an output energy of $300 \mathrm{keV} / \mathrm{amu}$. Operating at $200 \mathrm{MHz}$, the RFQ would require a vane voltage of $65 \mathrm{kV}$ and have a space charge limit of $10 \mathrm{~mA}$ (for gold ions in a charge state 45+, this limit would correspond to $10^{11}$ particles in a $100 \mu s$ injection interval). The structure is very compact, with a length of only $2.3 \mathrm{~m}$. The experience with RFQ accelerators so far has been excellent; they are very reliable machines requiring minimal maintenance and are simple to operate.

The next stage following the RFQ should be a linear accelerator. Recently new models have been developed, using individually phased superconducting cavities (21), (22), (23) and this technique can be applied for our purpose as well. References 21-23 describe the system operating at Argonne National Laboratory which will replace the negative ion source and a $9 \mathrm{MV}$ tandem; the BNL linac design, however, would be less critical because of a much higher input energy into the linac (at Argonne, the heavy ion source of the ECR-type is mounted on a high voltage platform and there is no RFQ in front of the 1 inac). The linac would accept the bunched beam from the RFQ and its transmission could be similarly very high (90\%). The accelerating voltage, in order to match the tandem irijector, should be around $15 \mathrm{MV}$, resulting again in a compact machine. 


\section{Conclusions and Recommendations}

At present there is no high charge state heavy ion source that could serve in an injector to replace the Tandem as the RHIC injector, especially for gold ions. Electron Beam Ion Sources have been developed for several applications, among them production of highly stripped or fully ionized particles for atomic physics studies (low intensity beams) and production of light and medium heavy ion beams for injection into synchrotrons (up to krypton) for fixed target studies. Several devices have been designed for fundamental studies of processes in sources of this type. The main advantages of an EBIS are the possibility of producing highly ionized particles in a beam with a very small emittance, a narrow charge state distribution, and, if designed and constructed properly, a reliable operation. On the other hand, so far achieved ion beam currents, especially of heavier ion species, are low and the tolerances in the design and fabrication extremely severe. Scaling rules for EBIS are simple and in the limited range of available parameters seem to agree with experimental results. Future development and expansion of the EBIS depends crucially on the development of high current, well confined electron beams (1-10 $\mathrm{x}$, up to $20 \mathrm{kev}$, similar to those in adivanced gyrotrons (24), with a good energy recovery. There is relatively little effort devoted to the design of an EBIS suitable for heavy ion collider applications, although many technologies required for such a device already exist, because RHIC is the first potential user world wide for such beams. With the project at CERN $>0$ accelerate heavy ions in LHC, there will be more stimulus for the development of high charge state sources in general and EBIS devices in particular.

Electron cyclotron resonance sources, in principle steady state devices, have mostly been developed and used as injectors into cyclotrons, where they perform reliably and very satisfactorily. The only source of this type serving as a synchrotron preinjector $(0, S)$ is the MINIMAFIOS at CERN. Its performance, however, was less satisfactory, (25) with large intensity variations. The 
main advantages of ECR sources are a simple design, not requiring tight tolerances and sophisticated technologies, and high intensity of beams if highly stripped particles are not needed. However, scaling rules of ECR sources are complex, involving plasma processes, and very empirical and approximative in nature. While it was advantageous for ECR operation at klystron frequencies to have the electron gun separated from the source itself and have it in the very reliable klystron tube instead, ECR scaling rules require extending the frequencies into the gyrotron range. Even with such an expansion of the frequency, accompanied by entering a new and costly technology, it is not certain that a source could be developed to satisfy the RHIC preinjector requirements.

It seems a program to study, design, and construct an EBIS source similar to the state-of-the-art device described earlier should be started with specific RHIC requirements in mind. There is a substantial expertise available at BNL in several aspects of an advanced EBIS device; however, advice and cooperation with outside experts will still be necessary. Development of an rf acceleratior (RFQ and a linac) is based on known technologies and should not present any great problem. 


\section{References}

1. E.D. Donets, Proc. Fitin All-Union Conf. on Charged Particle Accelerators, Voi. 1, Nauka, Moscow, 1977, p. 346.

2. V.0. Kostrciun, in AIP Conference Proceedings No. 188, p. 65.

3. J. Faure, et al., ibid., p. 102.

4. L. Liljeby and A. Engström, ibid., p. 27.

5. C.J. Herrlander, ibid., p. 379.

6. E.D. Donets, Nucl. Instr. Methods, Bg, 522 (1985).

7. R.W. Schmieder, et al., RSI 61, 259 (1990).

8. R. Becker, Ref. 2, p. 359.

9. Conceptual Design of RHIC, BNL Rep. 52195 (1989).

10. E.D. Donets, Sov. J. Part. Nucl. 13 (5), (1982), p. 387.

11. R.W. Schmieder, et al., Ref. 2, p. 45.

12. M*B. Schneider, et al., ibid, p. 158.

13. E.D. Donets, IEEE Trans. Nucl. Sci., Vol. NS-23, p. 897 (1976).

14. B.M. Penetrante, et al., Ref. 2, p. 145.

15. R. Geller, Proc. 1989 IEEE Part. Accel. Conf., Chicago, IL, p. 1088 .

16. C.M. Lyneis, T.A. Antaya, RSI 61 (1), 221 (1990).

17. G. Melin, et al., Journ. de Phys., Coll. C1, 50, p. C1-673 (1989).

18. W. Halverson, ibid., p. Cl-751.

19. R. Geller, CERN/PS 88-10, p. 10 (1988).

20. J. Staples, Ref. 2, p. 347 .

21. P.K. Den Hartog, et al., Ref. 15, p. 545.

22. K.W. Shepard, et al., ibid., p. 974.

23. L.M. Bolinger, et al., ibid, p. 1120.

24. R.J. Craig, et al., Proc. Int. Conf. on ECR Ion Sources and Their Applications, NSCL, Rep. \#MSVCP-47, p. 131 (1987).

25. R. Billinge, et al., CERN/PS 88-67, p. 12 (1988). 